\title{
Editorial
}

\section{Bifurcation and Chaos Theory in Electrical Power Systems: Analysis and Control}

\author{
Ahmad M. Harb, ${ }^{1}$ Issa Batarseh, ${ }^{2}$ \\ Lamine M. Mili, ${ }^{3}$ and Mohamed A. Zohdy ${ }^{4}$ \\ ${ }^{1}$ School of Natural Resources Engineering and Management, German Jordanian University (GJU), \\ Amman, Jordan \\ ${ }^{2}$ Electrical and Computer Engineering Department, University of Central Florida, Orlando, FL, USA \\ ${ }^{3}$ Department of Electrical and Computer Engineering, Virginia Tech., Falls Church, VA, USA \\ ${ }^{4}$ Department of Electrical and Computer Engineering, Oakland University, Rochester, MI, USA
}

Correspondence should be addressed to Ahmad M. Harb, aharb48@gmail.com

Received 27 August 2012; Accepted 27 August 2012

Copyright (C) 2012 Ahmad M. Harb et al. This is an open access article distributed under the Creative Commons Attribution License, which permits unrestricted use, distribution, and reproduction in any medium, provided the original work is properly cited.

Everywhere in our daily life we encounter nonlinear phenomena. In fact, it is well known that the real life is nonlinear. Because of difficulty of solving nonlinear problems, they used to go for easy way and linearize the problem based on some assumptions. These assumptions may lead to loss of important information in the system. Because of that, and to be more realistic, the objective of this special issue is to use the modern nonlinear theory (bifurcation and chaos) to establish real nonlinear models for practical systems, engineering sciences, and engineering applications.

The scope of this issue is to publish original papers on all topics related to nonlinear dynamics and its applications such as, power system, power electronics, electric machines, and renewable energy. The contributions concerned will be discussion of a practical problem, the formulating nonlinear model, and determination of closed form exact or numerical solutions.

In the past two decades, many researchers have investigated the modern nonlinear theory in electric circuits as well as power systems. In the paper "Nonlinear electrical circuit oscillator control based on backstepping method: a genetic algorithm approach," M. Khoeiniha et al. investigated the dynamics of nonlinear electrical circuit by means of modern nonlinear techniques and the control of a class of chaotic system by using backstepping method based on Lyapunov function. The behavior of such nonlinear system when they are under the influence of external sinusoidal disturbances with unknown amplitudes has been considered. The objective is to analyze the performance of this system at different amplitudes of disturbances. They illustrate the proposed approach for controlling of duffing oscillator 
problem to stabilize this system at the equilibrium point. Also Genetic Algorithm method (GAs) for computing the parameters of controller has been used. GAs can be successfully applied to achieve a better controller. Simulation results are shown the effectiveness of the proposed method.

In the paper "Phase and antiphase synchronization between 3-cell CNN and volta fractional order chaotic systems via active control," Z. Yaghoub et al. discussed and analyzed synchronization of fractional order chaotic dynamical in secure communications of analog and digital signals and cryptographic systems. They study the drive-response synchronization method for "phase and antiphase synchronization" of a class of fractional-order chaotic systems via active control method, using the 3-cell and volta systems as an example. These examples are used to illustrate the effectiveness of the synchronization method.

In the paper "Function projective synchronization of a class of chaotic systems with uncertain parameters," J. Guan investigates the function projective synchronization of a class of chaotic systems with uncertain parameters. Based on Lyapunov stability theory, the nonlinear adaptive control law and the parameter update law are derived to make the state of two chaotic systems function projective synchronized. Numerical simulations are presented to demonstrate the e ectiveness of the proposed adaptive scheme.

In the paper "A new four-scroll chaotic attractor consisted of transient chaotic two-scroll and ultimate chaotic two-scroll," Y. Xu et al. used the feedback controlling method to find a new four-scroll chaotic attractor. The novel chaotic system can generate four scrolls two of which are transient chaotic and the other two of which are ultimate chaotic. Of particular interest is that this novel system can generate one-scroll, two 2-scroll, and four-scroll with variation of a single parameter. We analyze the new system by means of phase portraits, Lyapunov exponents, fractional dimension, bifurcation diagram, and Poincaré map, respectively. The analysis results show clearly that this is a new chaotic system which deserves further detailed investigation.

In the paper "Lag synchronization of coupled multidelay systems," L. Qun et al. study the chaos synchronization, which is an active topic, and its possible applications. They presented an improved method for lag synchronization of chaotic systems with coupled multidelay. The Lyapunov theory is used to consider the sufficient condition for synchronization. The specific examples will demonstrate and verify the effectiveness of the proposed approach.

In the paper "Bifurcations in a generalization of the ZAD-technique: application to a DC$D C$ buck power converter," L. Torres et al. proposed a variation of ZAD technique, which is to extend the range of zero averaging of the switching surface (in the classic ZAD it is taken in a sampling period), to a number $K$ of sampling periods. This has led to a technique that has been named K-ZAD. Assuming a specific value for $K=2$, we have studied the 2ZAD technique. The latter has presented better results in terms of stability, regarding to the original ZAD technique. These results can be demonstrated in different state space graphs and bifurcation diagrams, which have been calculated based on the analysis done about the behavior of this new strategy.

Ahmad M. Harb

Issa Batarseh

Lamine M. Mili

Mohamed A. Zohdy 


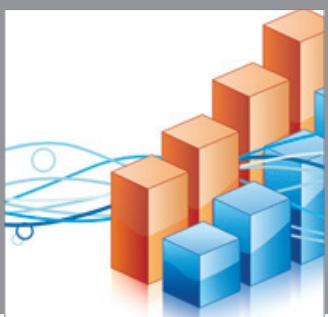

Advances in

Operations Research

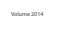

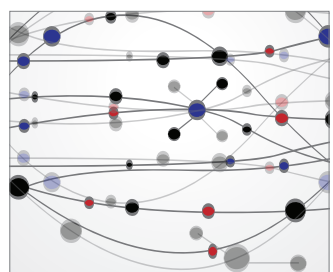

\section{The Scientific} World Journal
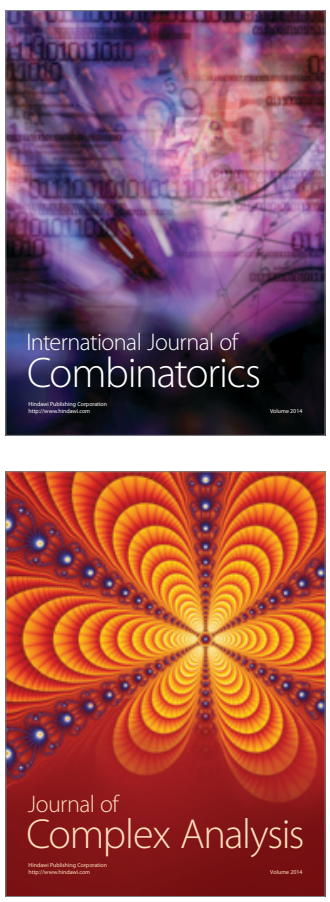

International Journal of

Mathematics and

Mathematical

Sciences
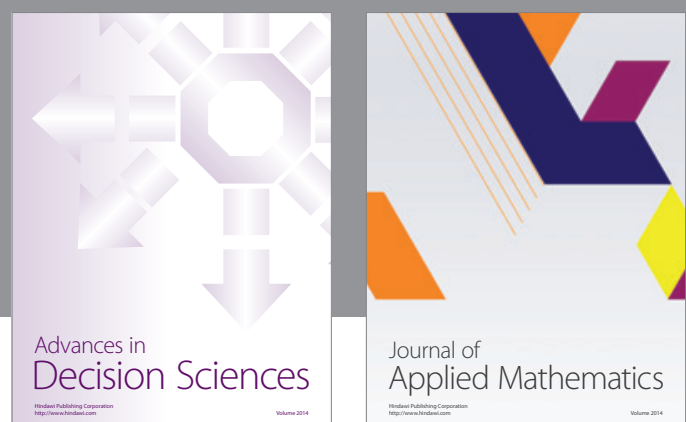

Journal of

Applied Mathematics
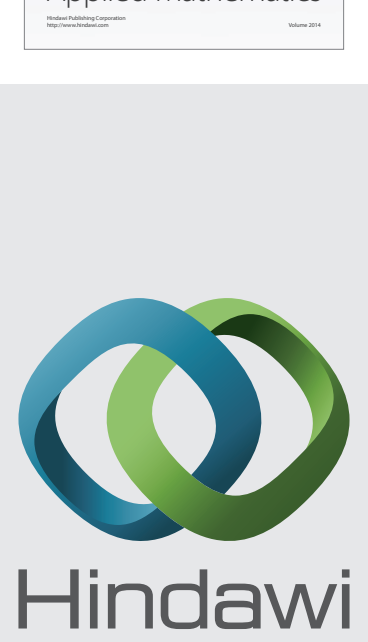

Submit your manuscripts at http://www.hindawi.com
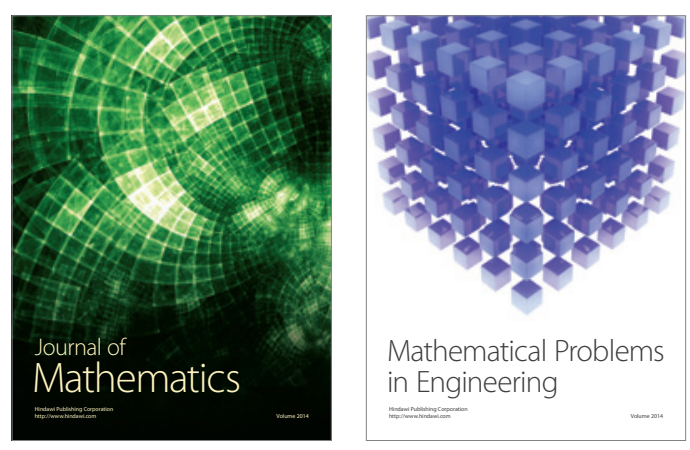

Mathematical Problems in Engineering
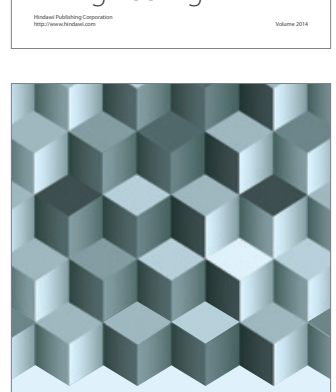

Journal of

Function Spaces
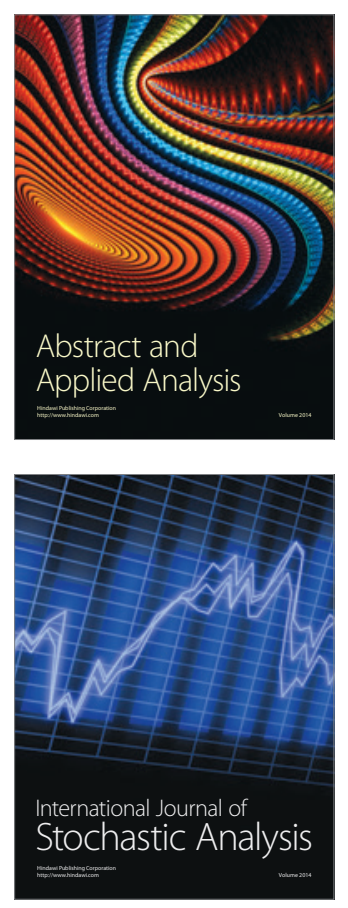

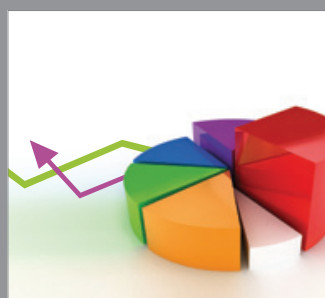

ournal of

Probability and Statistics

Promensencen
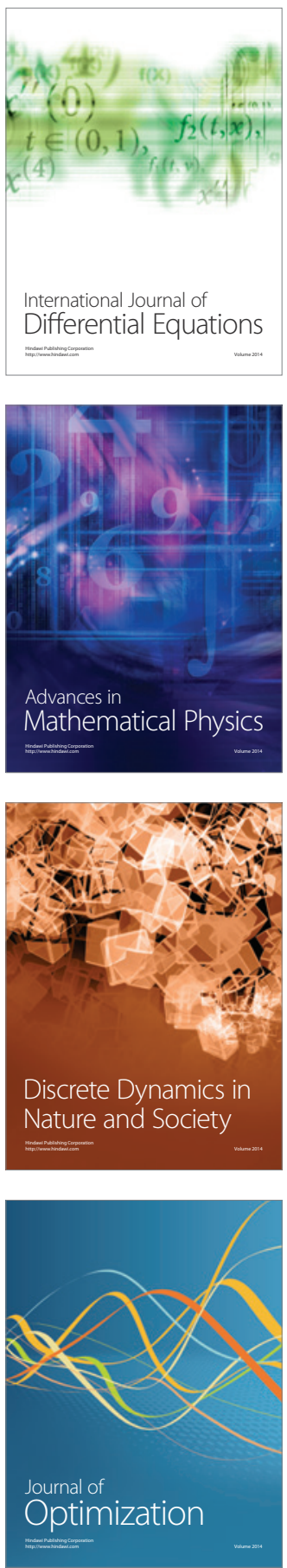